Éducation

et sociétés

plurilingues

\section{Éducation et sociétés plurilingues}

43 | 2017

Varia

\title{
Dario Elia TOSI, Diritto alla lingua in Europa
}

Giappichelli Editore, Torino, 2017, 416 p

\section{Luisa Revelli}

\section{(2) OpenEdition \\ Journals}

Edizione digitale

URL: http://journals.openedition.org/esp/1690

DOI: 10.4000/esp.1690

ISSN: 2532-0319

Editore

Centre d'Information sur l'Éducation Bilingue et Plurilingue

Edizione cartacea

Data di pubblicazione: 1 dicembre 2017

Paginazione: 93-95

ISSN: 1127-266X

Notizia bibliografica digitale

Luisa Revelli, «Dario Elia TOSI, Diritto alla lingua in Europa », Éducation et sociétés plurilingues [Online], 43 | 2017, Messo online il 01 février 2019, consultato il 24 septembre 2020. URL : http:// journals.openedition.org/esp/1690; DOI : https://doi.org/10.4000/esp.1690 


\section{GOMPTE-RENDU d'OUVRAGE}

\section{Dario Elia TOSI, Diritto alla lingua in Europa, Giappichelli Editore, Torino, 2017 [ISBN 978•88・9210856•1].}

\section{Luisa REVELLI}

Mots-clés: Droit à la langue, politique linguistique, minorisation linguistique, plurilinguisme en Europe

Keyzords: Language rights, linguistic policy, linguistic minorization, plurilingualism in Europe

Tl volume Dirillo alla lingua in Europa - pubblicato nei primi mesi dell'anno 2017 dall'editore Giappichelli di Torino all'interno della Collana «Le frontiere del Diritto» fondata da Giorgio Lombardi - affronta da un punto di vista giuridico e in prospettiva comparativa la questione delle lingue d'Europa esaminando scelte, indirizzi e provvedimenti che i diversi Paesi membri hanno adottato - nell'alveo collegiale della Comunità europea - come propri modelli di regolamentazione dei repertori linguistici nazionali.

L'Autore, docente di Diritto pubblico comparato presso il corso di laurea in Scienze Politiche e delle Relazioni Internazionali dell'Università della Valle d'Aosta, precisa preliminarmente che il suo studio non è dedicato a mere questioni di "diritto della lingua" ma si propone invece di esaminare le discipline normative relative al "diritto alla lingua" in un'ottica comprensiva tanto "dei diritti linguistici riconosciuti a determinati gruppi quanto [del]le prescrizioni sulla protezione delle lingue ufficiali poste a tutela degli interessi delle intere comunità statuali" (pag. XI). I dispositivi giuridici oggetto di osservazione riguardano pertanto sia i diritti sia i doveri: a una densa disamina storica dei modelli interpretativi degli uni e degli altri è rivolto il primo capitolo del volume, comprensivo di un excursus dedicato alle soluzioni terminologiche impiegate nel linguaggio settoriale giuridico per esprimere e rappresentare i rapporti fra lingue e diritto.

Sulle modalità attraverso le quali tali complessi rapporti sono stati assunti e affrontati nel corso del Novecento da parte della comunità internazionale si concentrano i due successivi capitoli: uno specifico approfondimento è dedicato ai contenuti della Convenzione Europea per la salvaguardia dei diritti dell'uomo e delle libertà 
Compte-rendu

L. Revelu fondamentali (GEDU) e alle loro applicazioni nella giurisprudenza della Corte di Strasburgo, con concreti esempi di pronunce in tema di tutela dei profili linguistici nazionali e di salvaguardia dei diritti dei cittadini appartenenti a comunità minoritarie.

La seconda parte del volume concerne l'analisi dei dispositivi di regolamentazione previsti negli ordinamenti nazionali dei 28 Paesi membri dell'Unione europea, dichiaratamente per sole ragioni espositive raggruppati nelle seguenti tre grandi macroaree: Europa Gentromeridionale; Europa centrosettentrionale; Europa centrorientale.

A ciascun Paese è dedicato uno specifico report, che confrontando i modelli di politica linguistica espressi negli ordinamenti legislativi con le concrete realtà dei repertori di riferimento mette in luce scelte politiche, implicazioni sociali, fenomeni di cambiamento, criticità e contraddizioni.

Nelle Conclusioni, Tosi osserva come il quadro delle discipline normative sul tema linguistico presenti caratteristiche di estrema variabilità: la comparazione fra le soluzioni adottate negli ordinamenti dei diversi Paesi dell'Unione evidenzia, infatti, un'ancora significativa eterogeneità delle politiche linguistiche nazionali, ciascuna indipendentemente dalle altre prodotto di implicite e intricate dinamiche storiche e sociali interne.

L'analisi delle tutele previste interiormente ai singoli Stati membri, d'altra parte, mette in luce marcate difformità nei confronti delle diverse minoranze compresenti, e il raffronto sugli spazi dedicati alle medesime parlate rappresentate in diverse aree geografiche manifesta eterogeneità rispetto all'impostazione di scelte e soluzioni. Così, accade in modo diffuso che coesistano spinti incoraggiamenti al pluralismo a fianco di misure protezionistiche a favore di lingue nazionali avvertite come deboli; che in Croazia gli italofoni godano di garanzie diverse da quelle concesse ai gruppi serbo-croati presenti nella medesima area; che i dispositivi di tutela concepiti per i parlanti catalani differiscano notevolmente a seconda che questi siano collocati in Spagna, Francia o Italia, e via discorrendo.

E, d'altra parte, osserva l'Autore che la progressivamente crescente consistenza dei flussi migratori verso l'Europa occidentale impone oggi l'individuazione di soluzioni capaci di rispondere efficacemente alla mutata conformazione degli assetti linguistici nazionali: seppur motivati da urgenze di integrazione degli immigrati con la comunità autoctona, i principi dell'assimilazionismo non appaiono rispondenti ai reali bisogni dei parlanti, per i quali l'appartenenza linguistica è portatrice di importanti implicazioni 
Compte-rendu

L. ReVELLI culturali, punti di riferimento valoriali e rappresentazioni identitarie.

In un quadro europeo che - conclude Tosi - "si presenterà in futuro sempre più postnazionale" i diversi sistemi politici dovranno quindi individuare le modalità più corrette per affrontare un inevitabile cambiamento anche al di là delle disposizioni normative, in quanto "continuare a governare il problema linguistico secondo gli stilemi tradizionali dello Stato nazione e dei rapporti maggioranze/minoranze appare riduttivo" (pag. 370). Con l'auspicio di un recupero "dell'impianto renaniano della nazione come frutto di un plebiscito di tutti i giorni", il modello proposto come auspicabile prospetta allora il superamento dei rigidi principi di tutela e protezione di lingue nazionali e minoranze, invocando invece l'adozione di ordinamenti politici che favoriscano il costituirsi di repertori plurilingui invece che multilingui; che ammettano aperture nei confronti degli idiomi delle comunità allofone delle nuove minoranze; che consentano, infine e in ogni caso, di "porre le basi per un processo osmotico in cui le singole identità si arricchiscano del contributo di ogni membro della comunità" con l'obiettivo conclusivo di "fare delle lingue un fattore di ricchezza, e non di divisione, dell'Unione" (pag. 371). 